\begin{tabular}{lc}
$\begin{array}{l}\text { Journal of Advanced } \\
\text { Engineering Trends }\end{array}$ & Vol.40, No.2. July 2021 \\
\hline ISSN : 26S2 - 2091 & http://jaet.journals.ekb.eg
\end{tabular}

\title{
Enhancement of Feed Forward Multi Effect Evaporator Performance for Water Desalination Using PI Control
}

\author{
BasmaKhairyAbdEl.Hakim $^{1}$, A. A. Abouelsoud ${ }^{2}$, Reda Abobeah ${ }^{1}$, Ebrahiem Esmail Ebrahiem $^{1}$ \\ ${ }^{I}$ Chemical Engineering Department, Faculty of Engineering, Minia University, Minia, Egypt \\ ${ }^{2}$ Electronics and Electrical Communications Engineering Department, Cairo University, Giza, Egypt \\ *Corresponding author E-mail:engbasma_khairy@mu.edu.eg
}

\begin{abstract}
The increasing of population needs safe, reliable and consistent supply of water had made many manufacturing industries and water treatment plants to look for efficient desalination plants. There are two major types of desalination technologies around the world, namely membrane desalination and thermal desalination . MEE is one of the types of thermal desalination. MED process operates in a series of evaporator condenser vessels called effects and uses the principle of reducing the ambient pressurein the various effects. A novel algorithm to solve the steady state analysis problem of three effects feed forward multi effect evaporator (FF MEE) for water desalination is investigated. A dynamic model is derived for MEE. FF MEE dynamic model is controlled using a Proportional-Integral (PI) controller that designed to improve its performance against the variation of the cold water temperature. Simulation results show the effectiveness of the proposed design and calculation has been presented using MATLAB ${ }^{\circledR}$.
\end{abstract}

Keywords: Feed forward multi effect evaporator- Water desalination-Steady state analysis-Dynamic model-PI controller

\section{INTRODUCTION}

Shortage of fresh water is a major problem affecting many countries. One of the ways to get an additional source of drinking water in places where there are much seawater resources is seawater desalination plants[1]. Population growth and industrial development have caused water shortage as a comprehensive crisis in many countries especially in the Middle East and North Africa [2]. Seawater desalination is very important technology to efficiently produce water for human use and irrigation from wastewater and seawater. Main desalination techniques are currently Multistage Flash Distillation (MSF), Multi-effect Distillation (MED) and Reverse osmosis(RO) [3]. Multi-effect desalination (MED) is the common technique that provides considerable quantity of potable water. This type of thermal desalination methods has been used recently because of its advantages such as low capital requirements, low operating costs, simple operating and maintenance procedures, thermal

Received:8Novamber, 2020, Accepted:23Novamber, 2020 efficiency, heat transfer coefficient, lower energy used and good performance ratio that is higher than other thermal desalination techniques like MSF[2] .

There are four different possible configurations for the MEE desalting systems, which differ in the flow directions of the heating steam and the evaporating brine, backward feed (BF), forward feed (FF), parallel feed (PF) and parallel/cross feed (PCF)[4]Transient modeling for different feed multi-effect evaporator (MEE)was investigated by a few researchers. For example, Miranda and Simpson [5] described a stationary and dynamic lumped model of backward feed MEE for tomato concentration. Tonelli et al. [6] presented an open-loop dynamic response model of triple effect evaporatorsfor apple juice concentrators with backward feed configuration.

Kumar et al. [7] modeled transient characteristics of mixed feed MEEsystem for paper industry based on the work in [8]. Their results showthat the effects temperature has a faster response compared to the solid concentration. The dynamic behavior of four effects parallel/cross MEDsystems was done by Aly and Marwan [8] which allowed the study ofsystem start-up, shutdown and load changes using lumped model ofmass, 
energy and salt balance equations. El-Nashar and Qamhiyeh [9]. The backward feed arrangement is not suitable for application in sea water desalination. The parallel feed layout is by no means the most economical and is efficient only when the feed brine is nearly saturated to boil inside the effects. The salt concentration reaches the maximum permissible value in all effects [10]. The aim of this paper is developing a dynamic modeling of MEE and improving its performance by using PI controller to eliminate the effect of disturbance.

\section{SYSTEM DESCRIPTION}

MED process operates in a series of evaporator condenser vessels called effects and uses the principle of reducing the ambient pressure in the various effects[2]. Sea water is fed to condenser then it is preheated to required temperature and then is forwarded to two streams; portion of the heated water is used as feed of evaporators and the other as cooling seawater is rejected back to the sea. The feed seawater is entered to the first effect and the steam also does that as a source of energy.

Part of the feed is evaporated and the produced vapor is used to evaporate feed in the next effect, the un-evaporated brine is fed to the next effect [11].Same change occurs in the $2^{\text {nd }}$ evaporator. Also, the process is repeated in $3^{\text {rd }}$ evaporator as shown in figure 1 .

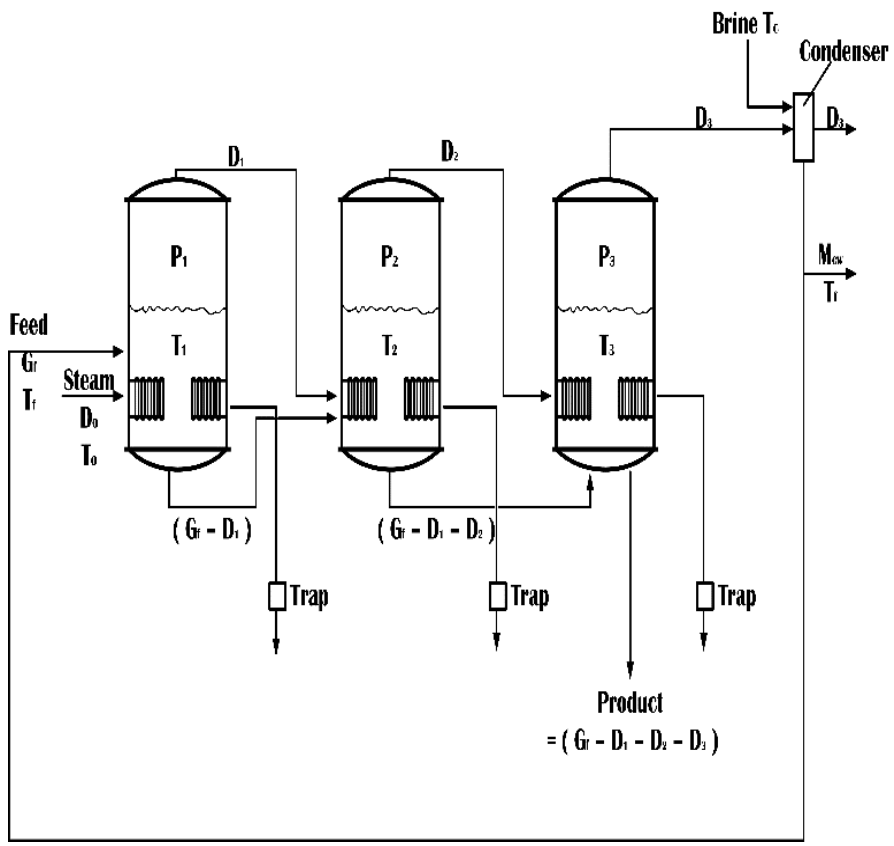

Figure 1: Three Effect Feed Forward MEE

\section{STEADY STATE ANALYSIS}

Brine solution has a boiling point greater than pure water depending in salt content and the difference between these two boiling points is called the Boiling Point Elevation (BPE). The variation of the boiling of saline solution with sodium chloride concentration can be estimated by an approximate relation

$$
T_{b}=T+a X
$$

Where,

$\boldsymbol{T}_{\boldsymbol{b}}$ : Boiling temperature of brine.

$\boldsymbol{T}$ : Boiling temperature of water (Given in steam tables)

$\boldsymbol{X}:$ Salt concentration in percent $\% .(\mathrm{kg} / \mathrm{kg})$

$\boldsymbol{a}:$ Coefficient $=0.05$ [12]

A recent formula for the saturation pressure of steam is given by [13] and results is shown in in figure 2 .

$$
P=P_{0} \exp \left[\frac{\left(A-B T+C T^{2}\right) T}{T+273}\right](2)
$$

Where $\boldsymbol{T}$ is temperature in ${ }^{\circ} \mathrm{C}$ with parameters $\boldsymbol{A}=19.846, \boldsymbol{B}=8.97 \times 10^{-3}, \boldsymbol{C}=1.248 \times 10^{-5}$ and $\boldsymbol{P}_{\boldsymbol{0}}=611.21 \mathrm{MPa}$ for temperature range from $0{ }^{\circ} \mathrm{C}$ to $110{ }^{\circ} \mathrm{C}$.

A formula for latent heat of vaporization of water as a function of temperature is given by [14] and results is shown in in figure 4.

$$
\begin{gathered}
\lambda=l-m T \\
l=2500.82 \frac{\mathrm{KJ}}{\mathrm{Kg}}, \quad m=2.358
\end{gathered}
$$

$\boldsymbol{T}$ is temperature in ${ }^{\circ} \mathrm{C}$.

This equation is valid for $0{ }^{\circ} \mathrm{C}$ to $50{ }^{\circ} \mathrm{C}$ but we shall use it up to $120{ }^{\circ} \mathrm{C}$ with negligible error $(15 / 2200) * 100 \%$ less than $1 \%$ as obtained from Figure 4. Table 1 gives the variation of saturation pressure and latent heat with temperature.

The effect of salt concentration $\boldsymbol{X}$ on $\boldsymbol{P}$ and latent heat was neglected. The partial derivatives of $\boldsymbol{T}_{\boldsymbol{b}}, \boldsymbol{P}$ and $\lambda$ are

$$
\frac{\partial T b}{\partial T}=1, \quad \frac{\partial T b}{\partial X}=a
$$

$\frac{\partial P}{\partial T}=P_{t}$

$=P \frac{\left(A-2 B T+3 C T^{2}\right)(T+273)-\left(A-B T+C T^{2}\right) T}{(T+273)^{2}}$ 


$$
\frac{\partial \lambda}{\partial T}=\lambda_{t}=-m
$$

The vapor density $\boldsymbol{\rho}_{\boldsymbol{v}}$ is calculated from pressure and temeprature by

$$
\rho_{v}=\frac{p}{R T}
$$

where $\boldsymbol{P}$ is calcualed from Eq. (2) and $\boldsymbol{R}_{w}$ is the gas constant for steam $(=461.52 \mathrm{~J} / \mathrm{kg} / \mathrm{K})$. The partial deriavtive of $\rho_{v}$ is

$$
\rho_{t}=\frac{\partial \rho_{v}}{\partial T}=\frac{P_{t}}{R_{w} T}-\frac{P}{R_{w} T^{2}}
$$

Maximum error $<1 \%$ at $120^{\circ} \mathrm{C}$ for three evaporators arranged as shown in Figure (4).

where the temperatures and pressures are $T_{1}, T_{2}, T_{3}$, and $\boldsymbol{P}_{\mathbf{1}}, \boldsymbol{P}_{\mathbf{2}}, \boldsymbol{P}_{\mathbf{3}}$ respectively, in each effect, if brine has noboiling point rise, then the heat transmitted per unit time across each effect is:

\section{Effect 1:}

$Q_{1}=U_{1} A_{1} \Delta T_{1}$, where $\Delta T_{1}=\left(T_{0}-T_{1}\right)$,

\section{Effect 2:}

$Q_{2}=U_{2} A_{2} \Delta T_{2}$, where $\Delta T_{2}=\left(T_{1}-T_{2}\right)$,

\section{Effect 3:}

$Q_{3}=U_{3} A_{3} \Delta T_{3}$, where $\Delta T_{3}=\left(T_{2}-T_{3}\right)$,

Neglecting the heat required to heat the feed from $\boldsymbol{T}_{f} \mathrm{to} \boldsymbol{T}_{\mathbf{1}}$, the heat $\boldsymbol{Q}_{\mathbf{1}}$ transferred across $\boldsymbol{A}_{1}$, assuming that the heat transferred is equal So :

$Q_{1}=Q_{2}=Q_{3}$

So that: $U_{1} A_{1} \Delta T_{1}=U_{2} A_{2} \Delta T_{2}=U_{3} A_{3} \Delta T_{3}$. If, as commonly the case, the individual effects are identical, $A_{1}=A_{2}=A_{3}$, and:

$U_{1} \Delta T_{1}=U_{2} \Delta T_{2}=U_{3} \Delta T_{3}$

The water evaporated in each effect is proportional to $Q$, since the latent heat is approximately constant. Thus the total capacity is:

$Q=Q_{1}+Q_{2}+Q_{3}$

$=U_{1} A_{1} \Delta T_{1}+U_{2} A_{2} \Delta T_{2}+U_{3} A_{3} \Delta T_{3}$

If an average value of the coefficients $\boldsymbol{U}_{\boldsymbol{a}}$ is taken, then:

$Q=U_{a v}\left(\Delta T_{1}+\Delta T_{2}+\Delta T_{3}\right) A$

Assuming the area of each effect is the same. At a pressure of $\boldsymbol{P}_{3} \mathrm{kN} / \mathrm{m}^{2}$, the boiling point of water is $\boldsymbol{T}_{3} \mathrm{~K}$, so that the total temperature difference $\Sigma \Delta T=T_{0}-T_{3} K$.
The latent heats $\lambda_{0}, \lambda_{1}, \lambda_{2}$ and $\lambda_{3}$, are givenin steam tables where :

$\lambda_{0} \mathrm{KJ} / \mathrm{Kgis}$ the latent heat at $\boldsymbol{T}_{\boldsymbol{0}}$ $\lambda_{1} \mathrm{KJ} / \mathrm{Kgis}$ the latent heat at $\boldsymbol{T}_{\boldsymbol{1}}$ $\lambda_{2} \mathrm{KJ} / \mathrm{Kgis}$ the latent heat at $\boldsymbol{T}_{2}$ $\lambda_{3} \mathrm{KJ} / \mathrm{Kgis}$ the latent heat at $\boldsymbol{T}_{3}$

Assuming that the condensate leaves at the steam temperature, and then heat balances across each effect may be made as follows:

\section{Effect 1:}

$D_{0} \lambda_{o}=G_{f} C_{p}\left(T_{1}-T_{f}\right)+D_{1} \lambda_{1}$

\section{Effect 2:}

$$
\left.\overline{D_{1} \lambda_{1}+\left(G_{f}\right.}-D_{1}\right) C_{p}\left(T_{1}-T_{2}\right)=D_{2} \lambda_{2} \text {, }
$$

\section{Effect 3:}

$D_{2} \lambda_{2}+\left(G_{f}-D_{1}-D_{2}\right) C_{p}\left(T_{2}-T_{3}\right)=D_{3} \lambda_{3}$,

Where $\boldsymbol{G}_{f}$ is the mass flow rate of brine fed to the system, and $\boldsymbol{C}_{\boldsymbol{p}}$ is the specific heat capacity of the liquid, which is assumed to be constant.

The material balance of sodium chloride gives $G_{f} X_{f}=\left(G_{f}-D_{1}-D_{2}-D_{3}\right) X_{3}$

That can put in the following matrix form:

$\left[\begin{array}{ccc}U_{1} & -U_{2} & 0 \\ 0 & U_{2} & -U_{3} \\ 1 & 1 & 1\end{array}\right]\left[\begin{array}{l}\Delta T_{1} \\ \Delta T_{2} \\ \Delta T_{3}\end{array}\right]=\left[\begin{array}{c}0 \\ 0 \\ T_{0}-T_{3}\end{array}\right]$

$$
\begin{gathered}
{\left[\begin{array}{cccc}
\lambda_{0} & -\lambda 1 & 0 & 0 \\
0 & \lambda_{1}-C_{p}\left(T_{1}-T_{2}\right) & -\lambda_{2} & 0 \\
0 & -C_{p}\left(T_{2}-T_{3}\right) & \lambda_{2}-C_{p}\left(T_{2}-T_{3}\right) & -\lambda_{3} \\
0 & X_{3} & X_{3} & X_{3}
\end{array}\right]} \\
{\left[\begin{array}{l}
D_{0} \\
D_{1} \\
D_{2} \\
D_{3}
\end{array}\right]=\left[\begin{array}{c}
G_{f} C_{p}\left(T_{1}-T_{f}\right) \\
-G_{f} C_{p}\left(T_{1}-T_{2}\right) \\
-G_{f} C_{p}\left(T_{2}-T_{3}\right) \\
G_{f}\left(X_{3}-X_{f}\right)
\end{array}\right]}
\end{gathered}
$$

First matrix equation gives $\Delta T_{1}, \Delta T_{2}$, and $\Delta T_{3}$ from which

$T_{1}=T_{0}-\Delta T_{1}, T_{2}=T_{1}-\Delta T_{2}$ and $T_{3}=T_{2}-\Delta T_{3}$

The second matrix equation gives $D_{0}, D_{1}, D_{2}$ and $\boldsymbol{D}_{3}$ To find $\boldsymbol{X}_{1}$ and $\boldsymbol{X}_{2}$ make material balance at the first effect and at the first and second effect respectively $G_{f} X_{f}=\left(G_{f}-D_{1}\right) X_{1}$

$G_{f} X_{f}=\left(G_{f}-D_{1}-D_{2}\right) X_{2}$

The heat balance at the condenser is:

$D_{3} \lambda_{3}=\left(G_{f}+M_{c w}\right) C_{p}\left(T_{f}-T_{c}\right)$

Having obtained $\boldsymbol{X}_{\mathbf{2}}$ and $\boldsymbol{X}_{\mathbf{3}}$ update the brine temperatures $\boldsymbol{T}_{\boldsymbol{b} \mathbf{3}}, \boldsymbol{T}_{\boldsymbol{b} \mathbf{2}}$ and $\boldsymbol{T}_{\boldsymbol{b} \mathbf{1}}$ 
Vol.40, No.2. July 2021

$$
\begin{gathered}
T_{b 3}=T_{3}+a X_{3} \\
T_{b 2}=T_{2}+a X_{2} T_{b 1}=T_{1}+a X_{1}
\end{gathered}
$$

As a check to the assumption of equal heat transfer area calculate

$A_{1}=D_{0} \lambda_{0} / U_{1} \Delta T_{1}$,

$A_{2}=D_{1} \lambda_{1} / U_{2} \Delta T_{2}$

$A_{3}=D_{2} \lambda_{2} / U_{3} \Delta T_{3}$

In the first iteration use $\boldsymbol{T}_{1}, \boldsymbol{T}_{2}$ and $\boldsymbol{T}_{3}$ in the second matrix Eq. In the subsequent iterations use $\boldsymbol{T} \boldsymbol{b}_{\boldsymbol{1}}, \boldsymbol{T} \boldsymbol{b}_{\boldsymbol{2}}$ and $\boldsymbol{T} \boldsymbol{b}_{3}$ in the second matrix Eq. Iterations are necessary to force $A_{1}=A_{2}=A_{3}$ approximately. $\boldsymbol{T}_{\boldsymbol{o}}$ update $\Delta T_{1}, \Delta T_{2}$, and $\Delta T_{3}$ as follows

$$
\Delta T_{(1)}=\Delta T_{(1)}+\left(A_{1}-A_{2}\right) / g
$$

$\Delta T_{(2)}=\Delta T_{(2)}+\left(A_{2}-A_{3}\right) / g(7)$

$$
\Delta \mathrm{T}_{(3)}=\Delta \mathrm{T}_{(3)}+\left(A_{3}-A_{1}\right) / g
$$

Table 1: Saturation steam pressure and latent heat from steam table

\begin{tabular}{|c|c|c|c|c|}
\hline $\mathbf{T}^{\circ} \mathbf{C}$ & $\mathbf{P} \mathbf{M P a}$ & $\boldsymbol{\lambda} \mathbf{K J} / \mathbf{K g}$ & $\mathbf{V}_{\mathbf{g}} \mathbf{~}^{\mathbf{3}} / \mathbf{K g}$ & $\boldsymbol{\rho}_{\mathbf{v}} \mathbf{K g} / \mathbf{m}^{\mathbf{3}}$ \\
\hline 20 & 0.002339 & 2453.5 & 57.76 & 0.0173 \\
\hline 25 & 0.003170 & 2441.7 & 43.34 & 0.0231 \\
\hline 30 & 0.004247 & 2429.8 & 32.88 & 0.0304 \\
\hline 35 & 0.005629 & 2417.9 & 25.21 & 0.0397 \\
\hline 40 & 0.007385 & 2406.0 & 19.52 & 0.0512 \\
\hline 45 & 0.009595 & 2394.0 & 15.25 & 0.0656 \\
\hline 50 & 0.01235 & 2382.0 & 12.03 & 0.0831 \\
\hline 55 & 0.01576 & 2369.8 & 9.564 & 0.1046 \\
\hline 60 & 0.01995 & 2357.6 & 7.667 & 0.1304 \\
\hline 65 & 0.02504 & 2345.4 & 6.194 & 0.1614 \\
\hline 70 & 0.03120 & 2333.0 & 5.040 & 0.1984 \\
\hline 75 & 0.03860 & 2320.6 & 4.129 & 0.2422 \\
\hline 80 & 0.04741 & 2308.0 & 3.405 & 0.2937 \\
\hline 85 & 0.05787 & 2295.3 & 2.826 & 0.3539 \\
\hline 90 & 0.07018 & 2282.5 & 2.359 & 0.4239 \\
\hline 95 & 0.08461 & 2269.5 & 1.981 & 0.5048 \\
\hline 100 & 0.1014 & 2256.4 & 1.672 & 0.5981 \\
\hline 110 & 0.1434 & 2229.7 & 1.209 & 0.8271 \\
\hline 120 & 0.1987 & 2202.1 & 0.8912 & 1.1221 \\
\hline
\end{tabular}




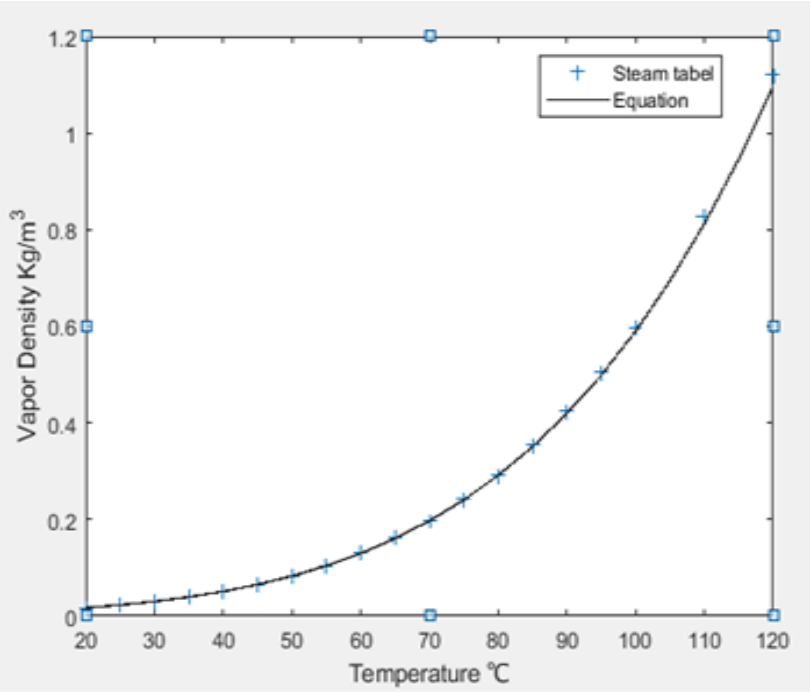

Figure 3: Measured and Calulated Vapor Density Eq. (4)

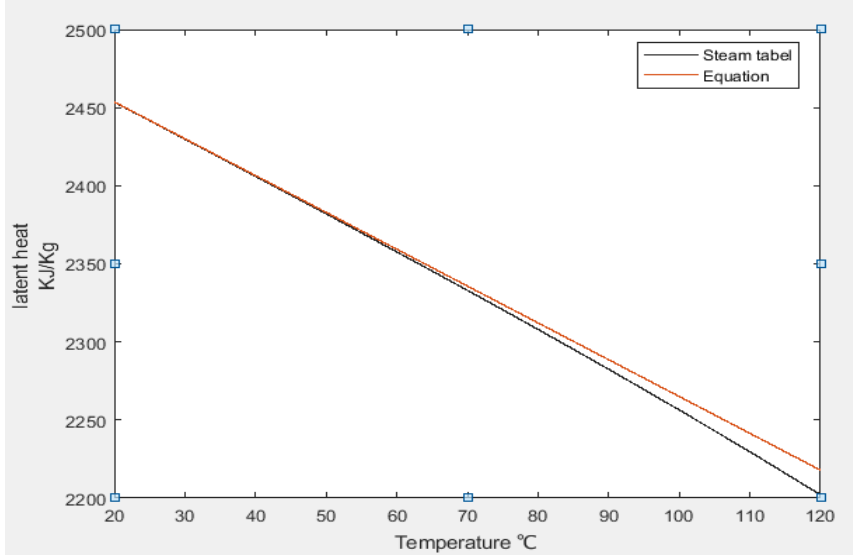

Figure 4: Latent Heat Variation with Temperature Eq. (3) and Calculations from Table (1)

\section{DYNAMIC MODEL OF THREE} EFFECTS FEED FORWARD MEE

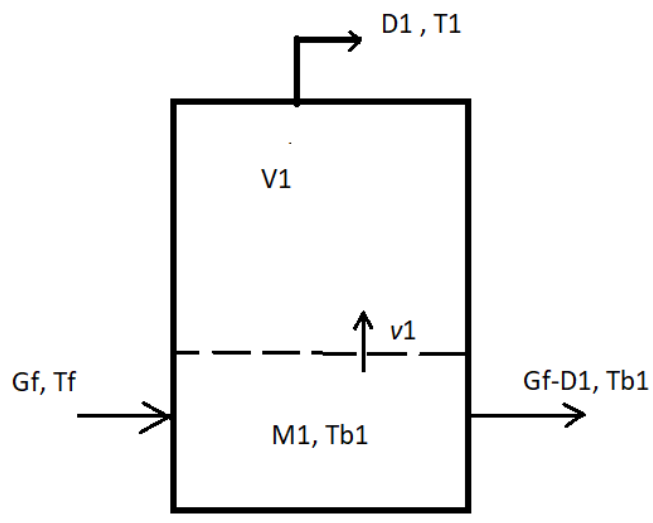

Figure 5: Fluid Components of First Effect
$M_{1}:$ Brine mass in effect $1 M_{1}=$ $\rho_{1} L_{1} \alpha$

$\boldsymbol{v}_{\boldsymbol{1}}$ : Vapor mass in from $\boldsymbol{M}_{\boldsymbol{1}}$ to $\boldsymbol{V}_{\boldsymbol{1}}$ effect 1

$\boldsymbol{V}_{\boldsymbol{l}}$ : vapor mass in effect $1 V_{1}=\rho_{v 1}\left(L-L_{1}\right) \alpha$

$\boldsymbol{L}:$ is the length of the effect $\alpha:$ is the effective area of the effect $\boldsymbol{L}_{\boldsymbol{l}}$ : is the brine level in the effect The enthalpy of $\boldsymbol{M}_{\boldsymbol{1}}$ :is $\rho_{1} L_{1} \alpha C_{p}\left(T_{b 1}-T_{R}\right)$ The enthalpy of $\boldsymbol{V}_{\boldsymbol{l}}:$ is $\rho_{v 1}\left(L-L_{1}\right) \alpha\left(C_{p}\left(T_{b 1}-\right.\right.$ $\left.\left.T_{R}\right)+\lambda_{1}\right)$

$\boldsymbol{T}_{R}$ : is a reference temperature $c_{l}$ coefficient of liquid discharge due to difference in liquid height Material and Energy balance of the first effect

\section{Liquid:}

$$
\begin{aligned}
& \frac{d M_{1}}{d t}=\frac{d\left(\rho_{1} L_{1} \alpha\right)}{d t} \\
& =G_{f}-v_{f}-\left(G_{f}-D_{1}\right)-c_{l}\left(L_{1}-L_{2}\right)
\end{aligned}
$$

Vapor:

Salt:

$$
\frac{d V_{1}}{d t}=\frac{d\left(\rho_{v 1}\left(L-L_{1}\right) \alpha\right)}{d t}=v_{1}-D_{1}
$$

$$
\frac{d X_{1} M_{1}}{d t}=\frac{d X_{1} \rho_{1} L_{1} \alpha}{d t}=X_{f} G_{f}-X_{1}\left(G_{f}-D_{1}\right):
$$

\section{Energy}

$\frac{d\left(\rho_{1} L_{1} \alpha C_{p}\left(T_{b 1}-T_{R}\right)+\rho_{v 1}\left(L-L_{1}\right) \alpha\left(C_{p}\left(T_{b 1}-T_{R}\right)+\lambda_{1}\right)\right)}{d t}$

$=D_{0} \lambda_{0}-D_{1} \lambda_{1}-C_{p}\left(G_{f}-D_{1}\right)\left(T_{b 1}-T_{f}\right)$

Adding first and second equations

$$
\frac{d\left(\alpha \rho_{1} L_{1}+\alpha \rho_{v 1}\left(L-L_{1}\right)\right)}{d t}=-c_{l}\left(L_{1}-L_{2}\right)
$$

Where $\frac{d \rho_{v 1}}{d t}=\frac{\rho_{t 1} d T_{1}}{d t}$

$$
\begin{gathered}
\left(\rho_{1}-\rho_{v 1}\right) \alpha \frac{d L_{1}}{d t}+\rho_{t 1} \alpha\left(L-L_{1}\right) \frac{d T_{1}}{d t} \\
=-c_{l}\left(L_{1}-L_{2}\right)
\end{gathered}
$$

Third Eq.

$$
\rho_{1} X_{1} \alpha \frac{d L_{1}}{d t}+\rho_{1} L_{1} \alpha \frac{d X_{1}}{d t}=X_{f} G_{f}-X_{1}\left(G_{f}-D_{1}\right)
$$

Fourth Eq.

$$
\begin{gathered}
\left\{\left(\rho_{1}-\rho_{v 1}\right) C_{p}\left(T_{b 1}-T_{R}\right)+\rho_{v 1} \lambda_{1}\right\} \alpha \frac{d L_{1}}{d t}+ \\
\left\{\left(\rho_{1} L_{1}+\rho_{v 1}\left(L-L_{1}\right)\right) C_{p} \alpha\right\} a \frac{d X_{1}}{d t}+
\end{gathered}
$$


Vol.40, No.2. July 2021

$$
\begin{aligned}
\left\{\left(\rho_{1} L_{1}+\rho_{v 1}(L-\right.\right. & \left.\left.L_{1}\right)\right) C_{p}+\left(L-L_{1}\right) C_{p}\left(T_{b 1}-T_{R}\right) \rho_{t 1} \\
& \left.-\rho_{v 1}\left(L-L_{1}\right) m\right\} \alpha \frac{d T_{1}}{d t} \\
& =D_{0} \lambda_{0}-D_{1} \lambda_{1}-C_{p}\left(G_{f}-D_{1}\right)\left(T_{b 1}-T_{f}\right)
\end{aligned}
$$

In matrix form

$$
\begin{aligned}
& {\left[\begin{array}{lll}
c_{11} & c_{12} & c_{13} \\
c_{21} & c_{22} & c_{23} \\
c_{31} & c_{32} & c_{33}
\end{array}\right]\left[\begin{array}{c}
\frac{d L_{1}}{d t} \\
\frac{d X_{1}}{d t} \\
\frac{d T_{1}}{d t}
\end{array}\right]} \\
& =\left[\begin{array}{c}
-c_{l}\left(L_{1}-L_{2}\right) \\
X_{f} G_{f}-X_{1}\left(G_{f}-D_{1}\right) \\
D_{0} \lambda_{0}-D_{1} \lambda_{1}-C_{p}\left(G_{f}-D_{1}\right)\left(T_{b 1}-T_{f}\right)
\end{array}\right]
\end{aligned}
$$

$c_{11}=\left(\rho_{1}-\rho_{v 1}\right) \alpha$

$c_{12}=0$

$c_{13}=\left(L-L_{1}\right) \alpha \rho_{t 1}$

$c_{21}=X_{1} \rho_{1} \alpha$,

$c_{22}=\rho_{1} L_{1} \alpha$

$$
c_{23}=0 \text {. }
$$

$$
\begin{gathered}
c_{31}=\left(\rho_{1}-\rho_{v 1}\right) C_{p}\left(T_{b 1}-T_{R}\right) \alpha-\rho_{v 1} \lambda_{1} \alpha, \\
c_{32}=\left(\rho_{1} L_{1}+\rho_{v 1}\left(L-L_{1}\right)\right) \alpha * C_{p} * a, \\
c_{33}=\left(\left(\rho_{1} L_{1}+\rho_{v 1}\left(L-L_{1}\right)\right) \alpha C_{p}\right. \\
+\left(L-\quad L_{1}\right) C_{p} \alpha\left(T_{b 1}\right. \\
\left.-T_{R}\right) \rho_{t 1}-\left(\alpha \rho_{v 1}\left(L-L_{1}\right) m\right.
\end{gathered}
$$

Calculated at $\boldsymbol{T}_{\boldsymbol{1 s s}}$ (steady state Temperature of first effect)

Eq. Can be written as

$$
E_{1}\left[\begin{array}{l}
\frac{d L_{1}}{d t} \\
\frac{d X_{1}}{d t} \\
\frac{d T_{1}}{d t}
\end{array}\right]=F_{1}
$$

Similarly for the second effect

$$
\left[\begin{array}{lll}
c_{11} & c_{12} & c_{13} \\
c_{21} & c_{22} & c_{23} \\
c_{31} & c_{32} & c_{33}
\end{array}\right]\left[\begin{array}{l}
\frac{d L_{2}}{d t} \\
\frac{d X_{2}}{d t} \\
\frac{d T_{2}}{d t}
\end{array}\right]=
$$

$$
\left[\begin{array}{c}
c_{l}\left(L_{1}-L_{2}\right)-c_{l}\left(L_{2}-L_{3}\right) \\
X_{1}\left(G_{f}-D_{1}\right)-X_{2}\left(G_{f}-D_{1}-D_{2}\right) \\
D_{1} \lambda_{1}-D_{2} \lambda_{2}-C_{p}\left(G_{f}-D_{1}-D_{2}\right)\left(T_{b 2}-T_{b 1}\right)
\end{array}\right](
$$

$c_{11}, \ldots c_{33}$ are the same as in Eqs.(9) but calculated at $\boldsymbol{T}_{2 s s}$ (steady state Temperature of second effect) For the third effect

$$
\begin{gathered}
{\left[\begin{array}{ccc}
c_{11} & c_{12} & c_{13} \\
c_{21} & c_{22} & c_{23} \\
c_{31} & c_{32} & c_{33}
\end{array}\right]\left[\begin{array}{c}
\frac{d L_{3}}{d t} \\
\frac{d X_{3}}{d t} \\
\frac{d T_{3}}{d t}
\end{array}\right]=} \\
{\left[\begin{array}{c}
c_{l}\left(L_{2}-L_{3}\right)-c_{l} L_{3} \\
X_{2}\left(G_{f}-D_{1}-D_{2}\right)-X_{3}\left(G_{f}-D_{1}-D_{2}-D_{3}\right) \\
D_{2} \lambda_{2}-D_{3} \lambda_{3}-C_{p}\left(G_{f}-D_{1}-D_{2}-D_{3}\right)\left(T_{b 3}-T_{b 2}\right)
\end{array}\right]}
\end{gathered}
$$

$c_{11}, \ldots c_{33}$ are the same as above but calculated at $\boldsymbol{T}_{3 s s}$ (steady state Temperature of third effect). Condenser dynamics

$C_{p} \rho_{f} V_{c} \frac{d T_{f}}{d t}=D_{3} \lambda_{3}-\left(G_{f}+M_{c w}\right)\left(T_{f}-T_{c}\right)$

$\boldsymbol{V}_{\boldsymbol{c}}, \boldsymbol{P}_{\boldsymbol{c}}$ are the condenser volume and pressure respectively. Eq.(9) Can be written as

$$
E_{1}\left[\begin{array}{l}
\frac{d L_{1}}{d t} \\
\frac{d X_{1}}{d t} \\
\frac{d T_{1}}{d t}
\end{array}\right]=F_{1}
$$

Similarly for Eq. (10) and Eq. (11)

Let

$$
\begin{aligned}
X^{T}= & {\left[L_{1}-L_{1 s} X_{1}-X_{1 s} T_{1}-T_{1 s} L_{2}-L_{2 s} X_{2}-X_{2 s}\right.} \\
& \left.T_{2}-T_{2 s} L_{3}-L_{3 s} X_{3}-X_{3 s} T_{3}-T_{3 s} T_{f}-T_{f s}\right]
\end{aligned}
$$

A relationship between $\boldsymbol{D}_{1}, \boldsymbol{D}_{2}$ and $\boldsymbol{D}_{3}$ and the temperatures $\boldsymbol{T}_{\mathbf{1}}, \boldsymbol{T}_{2}, \boldsymbol{T}_{\mathbf{3}}$

$$
\begin{gathered}
D_{1}=\gamma_{1}\left(P_{1}-P_{2}\right), D_{2}=\gamma_{2}\left(P_{2}-P_{3}\right), \\
D_{3}=\gamma_{3}\left(P_{3}-P_{c}\right)
\end{gathered}
$$

$\boldsymbol{P c}$ is the condenser pressure and $\boldsymbol{G}_{\boldsymbol{f}}$ and $\boldsymbol{X}_{\boldsymbol{f}}$ are constant. To find $\gamma_{1}$

$$
\begin{array}{r}
\gamma_{1}=\frac{D_{1 s}}{P_{1 s}-P_{2 s}} \\
\dot{x}=A x+b u+b d \quad(13)
\end{array}
$$


Vol.40, No.2. July 2021

$\boldsymbol{d}$ : Disturbance; change of $\boldsymbol{T}_{\boldsymbol{c}}$ from steady state $d=T_{c}-T_{c s}$

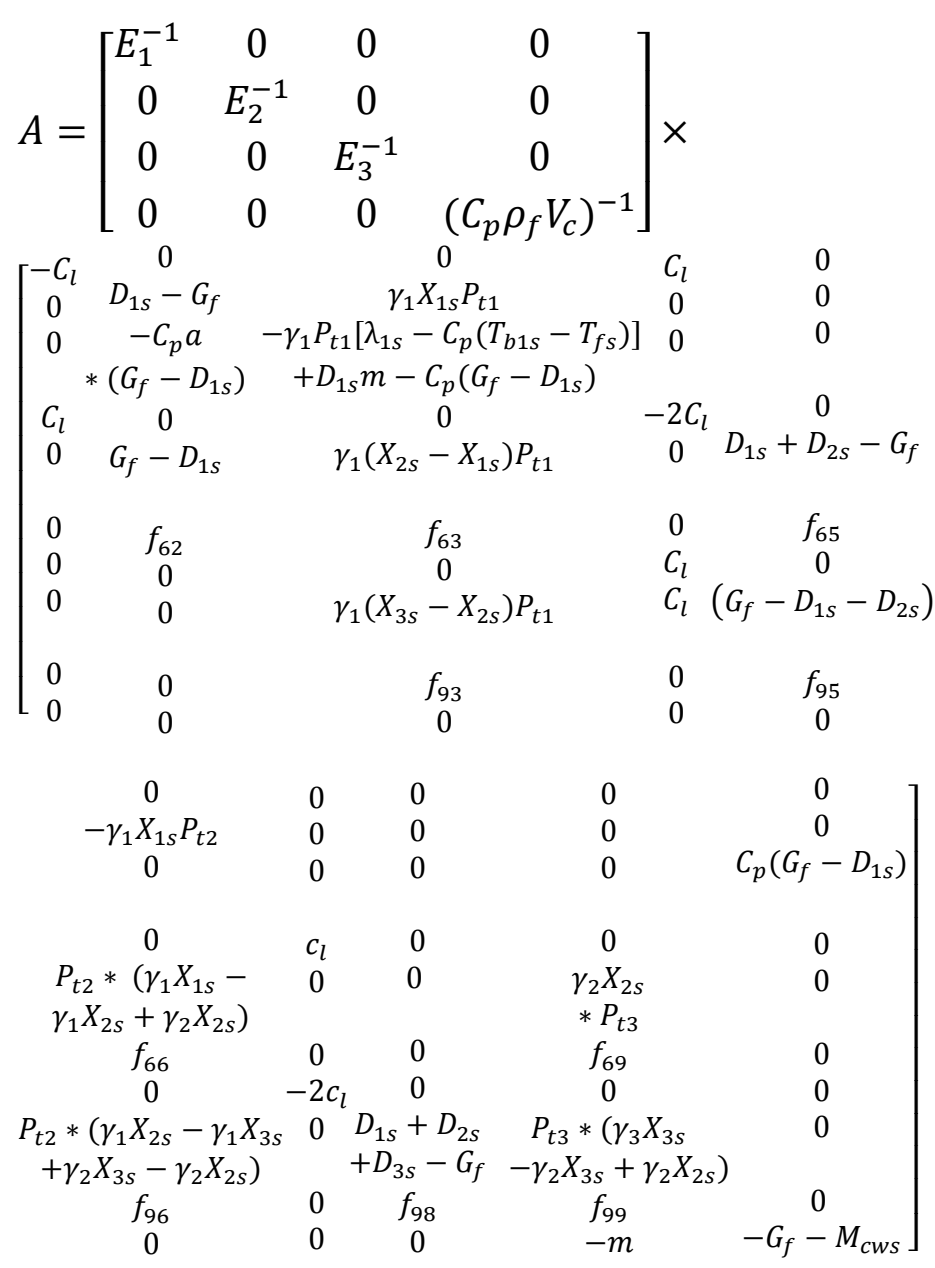

$f_{62}=C_{p}\left(G_{f}-D_{1 s}-D_{2 s}\right) a$

$f_{63}=-D_{1 s} m+\left(\lambda_{1 s}+C_{p}\left(T_{b 2}-T_{b 1}\right) \gamma_{1} P_{t 1}\right.$

$$
+C_{p}\left(G_{f}-D_{1 s}-D_{2 s}\right)
$$

$f_{65}=-f_{62}$

$f_{66}=D_{2 s} m-C_{p}\left(G_{f}-D_{1 s}-D_{2 s}\right)$

$+\left(-\lambda_{1 s}-C_{p}\left(T_{b 2}-T_{b 1}\right)\right) \gamma_{1}$

$-\left(\lambda_{2 s}-C_{p}\left(T_{b 2}-T_{b 1}\right) \gamma_{2} P_{t 2}\right.$

$f_{69}=\left(\lambda_{2 s}-C_{p}\left(T_{b 2}-T_{b 1}\right)\right) \gamma_{2} P_{t 3}$

$f_{93}=C_{p}\left(T_{b 3}-T_{b 2}\right) \gamma_{1} P_{t 1}$

$f_{95}=C_{p}\left(G_{f}-D_{1 s}-D_{2 s}-D_{3 s}\right) a$

$f_{96}=-D_{2 s} m+C_{p}\left(G_{f}-D_{1 s}-D_{2 s}-D_{3 s}\right)$

$$
\begin{aligned}
& +\left(\lambda_{2 s}+C_{p}\left(T_{b 3 s}-T_{b 2 s}\right)\right) \gamma_{2} \\
& \left.-C_{p}\left(T_{b 3 s}-T_{b 2 s}\right) \gamma_{1}\right) P_{t 2}
\end{aligned}
$$

$$
f_{98}=-f_{95}
$$

$f_{99}=D_{3 s} m-C_{p}\left(G_{f}-D_{1 s}-D_{2 s}-D_{3 s}\right)$

$$
+\left(-\gamma_{3} \lambda_{3}\right.
$$

$$
+\left(C_{p}\left(T_{b 3 s}-T_{b 2 s}\right) \gamma_{2}\right) P_{t 3}
$$

$$
b=\left[\begin{array}{cccc}
E_{1}^{-1} & 0 & 0 & 0 \\
0 & E_{2}^{-1} & 0 & 0 \\
0 & 0 & E_{3}^{-1} & 0 \\
0 & 0 & 0 & \left(C_{p} \rho_{f} V_{c}\right)^{-1}
\end{array}\right]\left[\begin{array}{c}
0 \\
0 \\
. . \\
0 \\
0 \\
T_{f S}-T_{c S}
\end{array}\right]
$$

\section{PERFORMANCE ENHACEMENT USING PI CONTROL}

A state feedback PI controller is used to eliminate the effect of disturbance $d\left(=T_{c w s}-T_{c W}\right)$ Choose an output of the system as the pressure of third effect $y=P_{3}$

$$
C=\left[\begin{array}{llllcccccc}
0 & 0 & 0 & 0 & 0 & 0 & 0 & 0 & P_{t 3} & 0
\end{array}\right],
$$
The third effect pressure $\boldsymbol{P}_{\boldsymbol{3}}$ is affected by the $u$ $\left(u=M_{c W}-M_{c w s}\right) \boldsymbol{D}$ is selected as a small number. Let $y_{R}=P_{3 R}$ be desired pressure of third effect

\section{Hence}

$$
p=\int\left(P_{3}-P_{3 R}\right) d t=\int\left(y-y_{R}\right) d t
$$

$$
\begin{gathered}
\dot{p}=C x-y_{R} \\
{\left[\begin{array}{c}
\dot{x} \\
\dot{p}
\end{array}\right]=\left[\begin{array}{cc}
A & 0 \\
C & 0
\end{array}\right]\left[\begin{array}{l}
x \\
p
\end{array}\right]+\left[\begin{array}{l}
b \\
0
\end{array}\right] u+\left[\begin{array}{cc}
b & 0 \\
0 & -1
\end{array}\right]\left[\begin{array}{c}
d \\
y_{R}
\end{array}\right]}
\end{gathered}
$$

At steady state $\dot{x}=\dot{p}=0$

$$
0=\left[\begin{array}{ll}
A & 0 \\
C & 0
\end{array}\right]\left[\begin{array}{l}
x_{s} \\
p_{s}
\end{array}\right]+\left[\begin{array}{l}
b \\
0
\end{array}\right] u_{s}+\left[\begin{array}{cc}
b & 0 \\
0 & -1
\end{array}\right]\left[\begin{array}{c}
d \\
y_{R}
\end{array}\right]
$$

Subtract these two equations

Let

$$
\left[\begin{array}{c}
\dot{x} \\
\dot{p}
\end{array}\right]=\left[\begin{array}{ll}
A & 0 \\
C & 0
\end{array}\right]\left[\begin{array}{l}
x-x_{s} \\
p-p_{s}
\end{array}\right]+\left[\begin{array}{l}
b \\
D
\end{array}\right]\left(u-u_{s}\right)
$$

$$
u-u_{s}=-k_{1}\left(x-x_{s}\right)-k_{2}\left(p-p_{s}\right)
$$

$\boldsymbol{k}_{\boldsymbol{1}}$ and $\boldsymbol{k}_{2}$ are selected to make the closed loop matrix have negative eigenvalues

$$
\left[\begin{array}{ll}
A & 0 \\
C & 0
\end{array}\right]-\left[\begin{array}{l}
b \\
0
\end{array}\right]\left[\begin{array}{ll}
k_{1} & k_{2}
\end{array}\right]
$$

The gain $\left[k_{1} k_{2}\right.$ ] can be obtained using lqr command The steady state terms in Eq. cancel and can be written as

$u=-k_{1} x-k_{2} p=-k_{1} x-k_{2} \int\left(y-y_{R}\right) d t$

Which is PI controller. Use lqr MATLAB command to design feedback controller. 


\section{SIMULATION RESULTS}

Two sub sections (static analysis section and closed loop analysis with feedback controller section) are considered here. In static analysis the design point that necessary to study the effect of deviation of the external input (e.g. $T c$ ) on the performance is calculated. This is carried out in subsection 2. Subsection 3 shows how the feedback Controller restores the performance using feedback control.

\section{a) Static analysis}

Table 2: The flowing data are used

\begin{tabular}{|l|l|l|l|l|}
\hline$T_{0}$ & $T_{f}$ & $P_{3}$ & $U_{I}$ & $U_{2}$ \\
$\mathbf{K}$ & $\mathrm{K}$ & $\mathrm{KN} / \mathbf{m}^{2}$ & $\mathrm{KW} / \mathbf{m}^{2} \mathbf{K}$ & $\mathbf{K W} / \mathbf{m}^{2} \mathbf{K}$ \\
\hline 394 & 294 & $\mathbf{1 3}$ & 3.1 & 2 \\
\hline$U_{3}$ & $X_{f}$ & $X_{3}$ & $\mathbf{C}_{\mathrm{p}}$ & $\mathbf{G}_{\mathbf{f}}$ \\
$\mathbf{K W} / \mathbf{m}^{2} \mathrm{~K}$ & $\%$ & $\%$ & $\mathrm{KJ} / \mathbf{K g ~ K}$ & $\mathbf{K g} / \mathbf{s}$ \\
\hline 1.1 & $\mathbf{1 0}$ & $\mathbf{5 0}$ & $\mathbf{4 . 1 8}$ & $\mathbf{4}$ \\
\hline
\end{tabular}

$T_{1}$ : is saturation temperature at $P_{3}=13 \mathrm{kN} / \mathrm{m}^{2}$ which equals $325 \mathrm{~K}$

Solving Eq. (1) with $\mathrm{T}_{0}-\mathrm{T}_{3}=394-325=69 \mathrm{~K}$,

Results of first iteration

$\Delta T_{1}=12.8535, \Delta T_{2}=19.9229, \quad \Delta T_{3}=36.2235$

$A_{1}=91.7003, A_{2}=55.1322, \quad A 3=61.4478$

Results of the $4^{\text {th }}$ iteration

$A_{1}=63.7015, A_{2}=65.1540, A_{3}=66.5189$

The vapor flow rates are

$D_{0}=1.6275, D_{l}=0.9914$,

$D_{2}=1.0680, D_{3}=1.1406$,

$D_{1}=0.9782$

$D_{2}=1.0679$

$D_{3}=1.1539$

The performance is $J=1.9662$

$A_{1}=91.7003, A_{2}=55.1322, A_{3}=61.4478$

Since areas are not equal increase $\Delta T_{l}$ and decrease

$\Delta T_{2}$ and $\Delta T_{3}$. To calculate the brine rejected $M_{c w}$ for

$T_{c}=288,=104.1583$

Another run

$G_{f}=4 ; T_{f}=320 ; T_{0}=394 ; T_{3}=325 ; T_{c}=298$

$A_{1}=61.9185, A_{2}=63.1129, A_{3}=64.1984$

$\Delta T_{1}=16.6170, \Delta T_{2}=17.6679, \quad \Delta T_{3}=34.7151$

$D_{0}=1.4397, D_{l}=0.9891, D_{2}=1.0676, D_{3}=1.1433$

$J=2.2227, M_{c w}=25.5674$

\section{b) Closed Loop Performance}

The closed loop gain is obtained using lqr command of MATLAB ${ }^{\circledR}$. Figure (6) shows the closed loop response for step input. The output is chosen to be the pressure of the third effect.

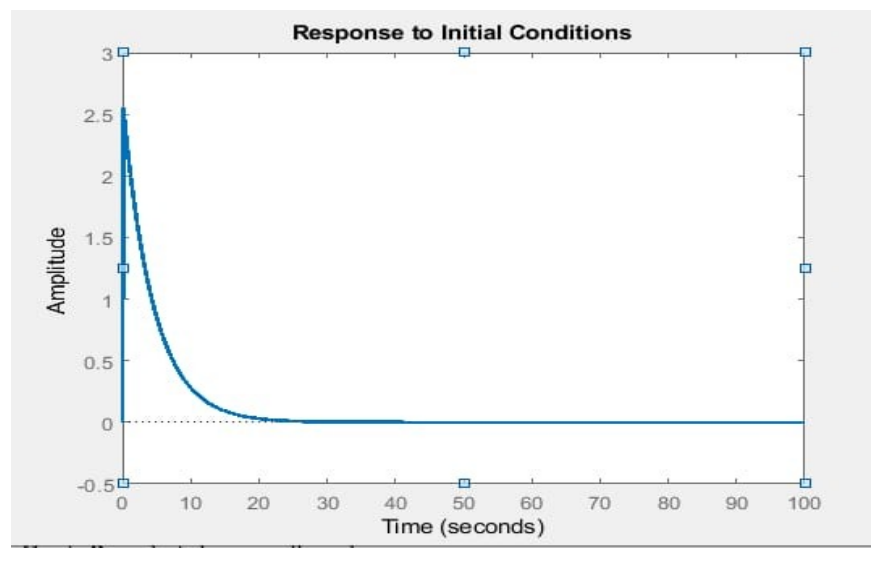

Figure 7 : Closed Loop Response with Output Pressure of the Third Effect

\section{CONCLUSION}

Novel algorithm for steady state calculation has been presented using MATLAB. At steady state, it was known the temperatures, pressures and flow rates of the three effects and performance of them. A dynamic model is derived for single effect then for MEE. PI controller has been designed to reject the effect of disturbance due to cold water temperature variation which degrades the performance of MEE system. Simulation part contains results of static analysis and feedback controller which restores the performance of the system. 


\section{REFERENCES}

[1] Roca, L., et al., Solar field control for desalination plants. Solar Energy, 2008. 82(9): p. 772-786.

[2] Mazini, M.T., A. Yazdizadeh, and M.H. Ramezani, Dynamic modeling of multi-effect desalination with thermal vapor compressor plant. Desalination, 2014. 353: p. 98-108.

[3] Lappalainen, J., T. Korvola, and V. Alopaeus, Modelling and dynamic simulation of a large MSF plant using local phase equilibrium and simultaneous mass, momentum, and energy solver. Computers \& Chemical Engineering, 2017. 97: p. 242258.

[4] Elsayed, M.L., et al., Transient performance of MED processes with different feed configurations. Desalination, 2018. 438: p. 37-53.

[5] V. Miranda, R. Simpson, Modelling and simulation of an industrial multiple effect evaporator: tomato concentrate, J. Food Eng. 66 (2) (2005) 203-210

[6] S.M. Tonelli, J. Romagnoli, J. Porras, Computer package for transient analysis of industrial multiple-effect evaporators, J. Food Eng. 12 (4) (1990) 267-281

[7] D. Kumar, V. Kumar, V. Singh, Modeling and dynamic simulation of mixed feed multi-effect evaporators in paper industry, Appl. Math. Model. 37 (1) (2013)384-397

[8] N.H. Aly, M. Marwan, Dynamic response of multi-effect evaporators, Desalination 114 (2) (1997) 189-196.
[9] A.M. El-Nashar, A. Qamhiyeh, Simulation of the performance of MES evaporators under unsteady state operating conditions, Desalination 79 (1) (1990) 65-83

[10] Hisham El-Dessouky, Imad Alatiqi, S. Bingulac and Hisham Ettouney" SteadyState Analysis of the Multiple Effect Evaporation Desalination Process" Chem. Eng. Technol. 21 (1998) 5

[11] Aly, N.H. and M. Marwan, Dynamic response of multi-effect evaporators. Desalination, 1997. 114(2): p. 189-196.

[12] Coulson and Richardson's “CHEMICAL ENGINEERING”, VOLUME 2,FIFTH EDITION butterworth and Heineman 2002.

[13] N. P. Romanov Izvestiya "A new formula for saturated water steam pressure within the temperature range -25 to $220^{\circ} \mathrm{C}^{\prime}$, Atmospheric and Oceanic Physics volume 45, pages799-804(2009)]

[14] B. Henderson-Sellers "A new formula for latent heat of vaporization of water as a function of temperature"Article in Quarterly Journal of the Royal Meteorological Society 110(466):1186 $1190 \cdot$ December 2006 


\section{Appendix A MATLAB script for steadystate}

clc

clear all

$\%$ data file name staticbasmaFF

$\mathrm{G}_{\mathrm{f}}=4 ; \mathrm{T}_{\mathrm{f}}=294 ; \mathrm{T}_{0}=394 ; \mathrm{T}_{3}=325 ; \mathrm{C}_{\mathrm{p}}=4.18 ; \mathrm{l}=2500.82 ; \mathrm{m}$ $=2.358$;

$\mathrm{U}_{1}=3.1 ; \mathrm{U}_{2}=2.0 ; \mathrm{U}_{3}=1.1 ; \mathrm{a}=0.05 ; \mathrm{X}_{3}=50 ; \mathrm{X}_{\mathrm{f}}=10 ;$

$\mathrm{M}_{1}=\left[\mathrm{U}_{1}-\mathrm{U}_{2} 0 ; 0 \mathrm{U}_{2}-\mathrm{U}_{3} ; 1 \mathrm{1} 1\right] ; \mathrm{B}_{1}=\left[0 ; 0 ; \mathrm{T}_{0}-\mathrm{T}_{3}\right]$;

$\mathrm{dT}=\operatorname{inv}\left(\mathrm{M}_{1}\right) * \mathrm{~B}_{1}$;

\section{$\%$ first iteration}

$\mathrm{T}_{1}=\mathrm{T}_{0}-\mathrm{dT}(1) ; \mathrm{T}_{2}=\mathrm{T}_{1}-\mathrm{dT}_{(2)} ; \mathrm{T}_{3}=\mathrm{T}_{2}-\mathrm{dT}_{(3)} ;$

$1 \mathrm{t}_{0}=1-\mathrm{m} *\left(\mathrm{~T}_{0^{-}}-273\right) ; \mathrm{lt}_{1}=1-\mathrm{m}^{*}\left(\mathrm{~T}_{1}-273\right) ; \mathrm{lt}_{2}=1-\mathrm{m}^{*}\left(\mathrm{~T}_{2^{-}}\right.$

273); $1 \mathrm{t} 3=1-\mathrm{m} *\left(\mathrm{~T}_{3}-273\right)$;

$\mathrm{M}_{2}=\left[\mathrm{lt}_{0}-\mathrm{lt}_{1} \mathbf{0} \quad 0 ; 0 \mathrm{lt}_{1}-\mathrm{C}_{\mathrm{p}} *\left(\mathrm{~T}_{1}-\mathrm{T}_{2}\right)-\mathrm{lt}_{2} 0\right.$;

$\left.0-\mathrm{C}_{\mathrm{p}} *\left(\mathrm{~T}_{2}-\mathrm{T}_{3}\right) \mathrm{lt}_{2}-\mathrm{C}_{\mathrm{p}} *\left(\mathrm{~T}_{2}-\mathrm{T}_{3}\right)-\mathrm{lt}_{3} ; 0 \mathrm{X}_{3} \mathrm{X}_{3} \mathrm{X}_{3}\right]$;

$\mathrm{B}_{2}=\left[\mathrm{G}_{\mathrm{f}} * \mathrm{C}_{\mathrm{p}} *\left(\mathrm{~T}_{1}-\mathrm{T}_{\mathrm{f}}\right) ;-\mathrm{G}_{\mathrm{f}} * \mathrm{C}_{\mathrm{p}} *\left(\mathrm{~T}_{1}-\mathrm{T}_{2}\right) ;-\mathrm{G}_{\mathrm{f}} * \mathrm{C}_{\mathrm{p}} *\left(\mathrm{~T}_{2}-\right.\right.$

$\left.\left.\mathrm{T}_{3}\right) ; \mathrm{G}_{\mathrm{f}}^{*}\left(\mathrm{X}_{3}-\mathrm{X}_{\mathrm{f}}\right)\right]$

$\mathrm{D}=\operatorname{inv}\left(\mathrm{M}_{2}\right) * \mathrm{~B}_{2} ; \mathrm{D}_{0}=\mathrm{D}_{(1)} ; \mathrm{D}_{1}=\mathrm{D}_{(2)} ; \mathrm{D}_{2}=\mathrm{D}_{(3)} ; \mathrm{D}_{3}=\mathrm{D}_{(4)}$;

$\mathrm{X}_{1}=\mathrm{G}_{\mathrm{f}} * \mathrm{X}_{\mathrm{f}} /\left(\mathrm{G}_{\mathrm{f}}-\mathrm{D}_{1}\right) ; \mathrm{X}_{2}=\mathrm{G}_{\mathrm{f}} * \mathrm{X}_{\mathrm{f}} /\left(\mathrm{G}_{\mathrm{f}}-\mathrm{D}_{1}-\mathrm{D}_{2}\right)$;

$\mathrm{Tb}_{1}=\mathrm{T}_{1}+\mathrm{a} * \mathrm{X}_{1} ; \mathrm{Tb}_{2}=\mathrm{T}_{2}+\mathrm{a} * \mathrm{X}_{2} ; \mathrm{Tb}_{3}=\mathrm{T}_{3}+\mathrm{a} * \mathrm{X}_{3}$;

$\mathrm{dT}$;

$\mathrm{J}=\left(\mathrm{D}_{1}+\mathrm{D}_{2}+\mathrm{D}_{3}\right) / \mathrm{D}_{0}$;

$\mathrm{A}_{1}=\mathrm{D}_{0} * 1 \mathrm{t}_{0} / \mathrm{U}_{1} / \mathrm{dT}_{(1)}, \mathrm{A}_{2}=\mathrm{D}_{1} * \mathrm{lt}_{1} / \mathrm{U}_{2} / \mathrm{dT}_{(2)}, \mathrm{A}_{3}=\mathrm{D}_{2} * 1 \mathrm{t}_{2} /$ $\mathrm{U}_{3} / \mathrm{dT}_{(3)}$

\section{\%loop iteration}

fori $=1: 3$

$\mathrm{g}=10$;

$\mathrm{dT}_{(1)}=\mathrm{dT}_{(1)}+\left(\mathrm{A}_{1}-\mathrm{A}_{2}\right) / \mathrm{g} ; \mathrm{dT}_{(2)}=\mathrm{dT}_{(2)}+\left(\mathrm{A}_{2}-\mathrm{A}_{3}\right) / \mathrm{g}$

$; \mathrm{dT}(3)=\mathrm{dT}_{(3)}+\left(\mathrm{A}_{3}-\mathrm{A}_{1}\right) / \mathrm{g}$;

$\mathrm{T}_{1}=\mathrm{T}_{0}-\mathrm{dT}_{(1)} ; \mathrm{T}_{2}=\mathrm{T}_{1}-\mathrm{dT}_{(2)} ; \mathrm{T}_{3}=\mathrm{T}_{2}-\mathrm{dT}_{(3)}$;

$\mathrm{lt}_{0}=1-\mathrm{m} *\left(\mathrm{~T}_{0}-273\right) ; \mathrm{lt}_{1}=1-\mathrm{m} *\left(\mathrm{~T}_{1}-273\right) ; \mathrm{lt}_{2}=1-\mathrm{m}^{*}\left(\mathrm{~T}_{2}-\right.$

$273) ; \mathrm{lt}_{3}=1-\mathrm{m}^{*}\left(\mathrm{~T}_{3}-273\right)$;

$0 \mathrm{M}_{2}=\left[\mathrm{lt}_{0}-\mathrm{lt}_{1} \mathbf{0} \quad 0 ; 0 \mathrm{lt}_{1}-\mathrm{C}_{\mathrm{p}} *\left(\mathrm{~T}_{\mathrm{b} 1}-\mathrm{T}_{\mathrm{b} 2}\right)-\mathrm{lt}_{2} 0 ; 0-\right.$

$\left.\mathrm{C}_{\mathrm{p}} *\left(\mathrm{~Tb}_{2}-\mathrm{Tb}_{3}\right) \mathrm{lt}_{2}-\mathrm{C}_{\mathrm{p}} *\left(\mathrm{~T}_{\mathrm{b} 2}-\mathrm{T}_{\mathrm{b} 3}\right)-\mathrm{lt}_{3} ; 0 \mathrm{X}_{3} \mathrm{X}_{3} \mathrm{X}_{3}\right]$;

$\mathrm{B}_{2}=\left[\mathrm{G}_{\mathrm{f}} * \mathrm{C}_{\mathrm{p}} *\left(\mathrm{~T}_{1}-\mathrm{T}_{\mathrm{f}}\right) ;-\mathrm{G}_{\mathrm{f}} * \mathrm{C}_{\mathrm{p}} *\left(\mathrm{~T}_{\mathrm{b} 1}-\mathrm{T}_{\mathrm{b} 2}\right) ;-\mathrm{G}_{\mathrm{f}} * \mathrm{C}_{\mathrm{p}} *\left(\mathrm{~T}_{\mathrm{b} 2}-\right.\right.$ $\left.\left.\mathrm{T}_{\mathrm{b} 3}\right) ; \mathrm{G}_{\mathrm{f}} *\left(\mathrm{X}_{3}-\mathrm{X}_{\mathrm{f}}\right)\right]$;

$\mathrm{D}=\operatorname{inv}\left(\mathrm{M}_{2}\right) * \mathrm{~B}_{2} ; \mathrm{D}_{0}=\mathrm{D}_{(1)} ; \mathrm{D}_{1}=\mathrm{D}_{(2)} ; \mathrm{D}_{2}=\mathrm{D}_{(3)} ; \mathrm{D}_{3}=\mathrm{D}_{(4)}$;

$\mathrm{X}_{1}=\mathrm{G}_{\mathrm{f}} * \mathrm{X}_{\mathrm{f}} /\left(\mathrm{G}_{\mathrm{f}}-\mathrm{D}_{1}\right) ; \mathrm{X}_{2}=\mathrm{G}_{\mathrm{f}} * \mathrm{X}_{\mathrm{f}} /\left(\mathrm{G}_{\mathrm{f}}-\mathrm{D}_{1}-\mathrm{D}_{2}\right)$;

$\mathrm{T}_{\mathrm{b} 1}=\mathrm{T}_{1}+\mathrm{a} * \mathrm{X}_{1} ; \mathrm{T}_{\mathrm{b} 2}=\mathrm{T}_{2}+\mathrm{a} * \mathrm{X}_{2} ; \mathrm{T}_{\mathrm{b} 3}=\mathrm{T}_{3}+\mathrm{a} * \mathrm{X}_{3}$;

dT;

$\mathrm{J}=\left(\mathrm{D}_{1}+\mathrm{D}_{2}+\mathrm{D}_{3}\right) / \mathrm{D}_{0}$

$\mathrm{A}_{1}=\mathrm{D}_{0} * 1 \mathrm{t}_{0} / \mathrm{U}_{1} / \mathrm{dT}_{(1)}, \mathrm{A}_{2}=\mathrm{D}_{1} * 1 \mathrm{t}_{1} / \mathrm{U}_{2} / \mathrm{dT}_{(2)}, \mathrm{A}_{3}=\mathrm{D}_{2} * 1 \mathrm{t}_{2} /$ $\mathrm{U}_{3} / \mathrm{dT}_{(3)}$,

end 


\section{Vol.40, No.2. July 2021}

\section{تحسين الأداء لنظام متعدد مراحل المبخرات لتحلية المياه باستخدام نظام التحكم PI}

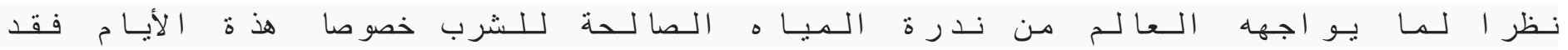

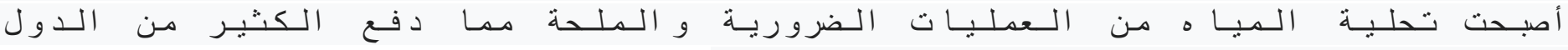

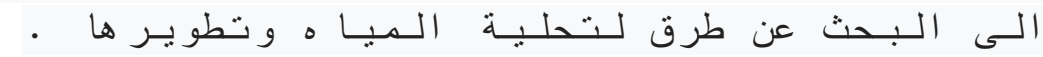

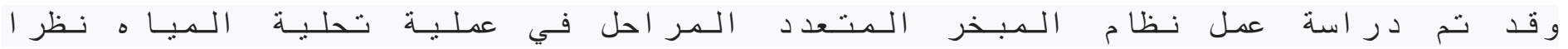

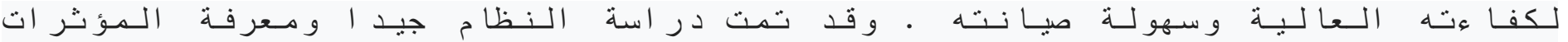

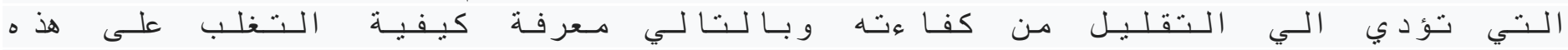

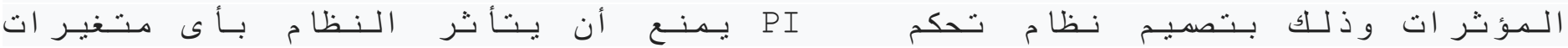

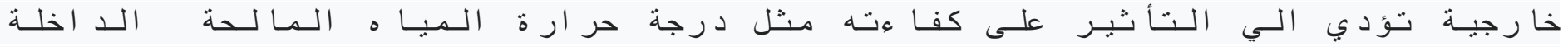

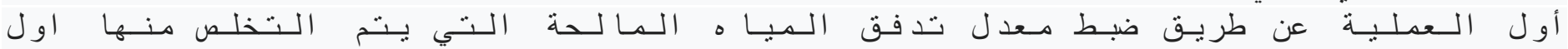

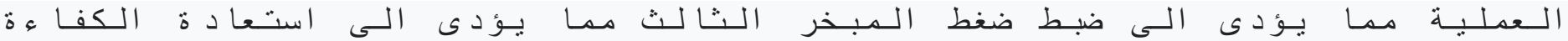

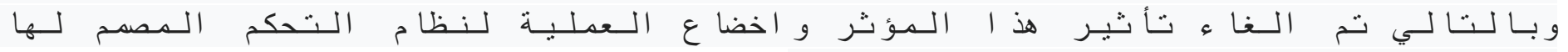

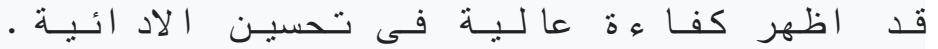

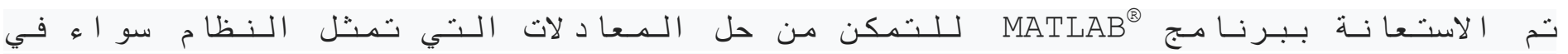

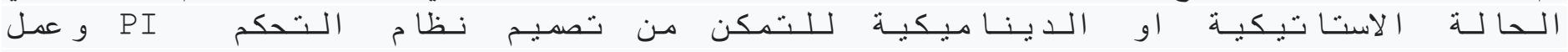

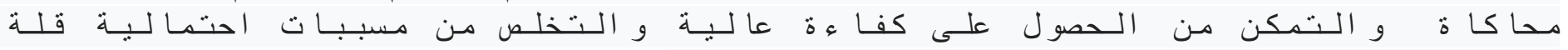

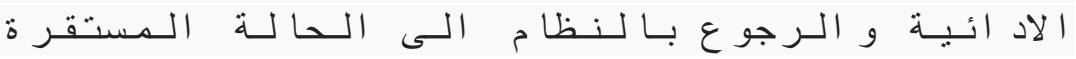

